\title{
Blood-Brain Barrier Disruption Chemotherapy
}

National Cancer Institute

\section{Source}

National Cancer Institute. Blood-Brain Barrier Disruption Chemotherapy. NCI Thesaurus.

Code $C 16026$.

Treatment modality used to increase the delivery of chemotherapeutic agents and monoclonal antibodies to the central nervous system tissue through temporal disruption of the blood brain barrier. 